\title{
HIGH-PRECISION DEM PRODUCTION FOR SPACEBORNE STEREO SAR IMAGES BASED ON SIFT MATCHING AND REGION-BASED LEAST SQUARES MATCHING
}

\section{Shi Xiaotian ${ }^{\text {a }}$, Zhang Guo ${ }^{\mathrm{a}, \mathrm{b}}$, Wang xia ${ }^{\mathrm{b}}$}

a. State key laboratory of information engineering in surveying, mapping and remote sens ing of Wuhan University, Hubei 430079, China;

b. Satellite of Surveying and Mapping Application center, State Bu reau of Surveying and Mapping, Beijing 100830, China

\section{Commission I, WG: I/2}

KEYWORDS : Matching, Stereo, DEM, Radargrammetric, Apply cation strategy.

\section{ABS TRACT:}

Generally, there are two ways to generate Digital Elev ation Model (DEM) using synthetic aperture radar (SAR) data, which are Interferometer Sy nthetic Aperture Radar(InSAR) and radargrammetry. Considering the disadvantages of InSAR data, such as the limit of terrain and the influence of water content, the application field of InSAR is relatively limited, while rad argrammetry is more widely applied since it does not have such limits. However, for high-precision stereo SAR imagery, since the terrain distortion caused by shooting angle cannot be eliminated and the speckle noises are obvious, the classical matching algorithms for optical stereo images do not have the same effect on SAR data.

Based on the experience of optical stereo image matching, this paper proposes a new algorithm which combines the feature of SIFT image matching, region-based least squares matching and TIN. First, SIFT matching is used as the initial matching to obtain the sparse DEM, then by using TIN the matching points are forecast, finally the region-based least squares matching is adopted to get accurate matching points. In this paper, COSMO-SkyMed and TSX stereo images of Lanzhou area are used to validate the proposed method. Experiment results show that the algorithm can be effectively used in stereo SAR matching and high-precision DEM production.

\section{INTRODUCTION}

Generally speaking, in remoting sensing, there are 3 ways to get the terrain information of the earth. They are Navigation Satellite Timing And Ranging Global Position System (GPS), Synthetic Aperture Radar (SAR) and Light Detection and Ranging (LiDAR).

Among them, Synthetic Aperture Radar (SAR) is special for its feature as can be used in all weather, all time and is strong permeability. With those advantages, SAR is used as an important data for extracting the height. While InSAR has its special needs in data in order to avoid the decorrelation between the two images, radargrammetry does not require so much as InSAR does. So radargrammetry can be used more flexibly. But radargrammetry has its own problem that needed to be solved. In geometries, mainly owning to the big change in nadir angle and different height of terrain, stereo images have different geometric deformation. And owning to the influence of speckle nose, some feature of terrain may be covered. So it is hard to match two SAR stereo images.

In optical image matching, SIFT matching and Region-Based least squares matching have been proved to be feasible and effective, But SAR image is different. Because the influence carried by speckle nose, if we simply using SIFT matching, then we can not get the result which is good enough. As we texted in the past, the points in SAR stereo images get by SIFT matching is accurate. But the points are not dense enough and are not distributed. So when we using those corresponding image points to get the height of earth by forward intersection 
and using TIN to product DEM, it has some problems, such as large area without data and the low accuracy. Region-Based least squares matching is reliable, accuracy and flexibly in theory, But compare with SIFT matching Region-Based least squares matching needs initial height to iterative calculus. Here based on the advantage of SIFT matching and Region-Based least squares matching and the success of the application of those two algorithms in optical image we figure a new way to matching stereo SAR images which can avoid the shortage of those two algorithm. That is using SIFT matching for initial matching, then add points and using TIN to forecast the match point. Using the initial corresponding image points to calculate the initial height, and then using the initial height in Region-Based least squares matching to calculate the right corresponding image point.

\section{ALGORITHM INTRODUCTION}

\section{- 2.1 SIFT MATCHING}

SIFT(scale invariant feature transform) is a algorithm that can transform an image into a large collection of local feature vectors which are invariant to image translation, scaling and rotation(Lowe, 1999). So in the same area, the same SIFT keypoints exacted from images stand for the same terrain stably. In SIFT matching there are 2 steps which are extract the keypoints and describe, match the keypoints.

\subsubsection{The EXTRACTION OF SIFT KEYPOINTS $x$}

In SIFT matching we need to extract the keypoints which are invariant to image translation, scaling and rotation. In order to computer and extract the keypoint efficiently, we use the difference of Gaussian function(Lowe, 2004). E.g.

$$
\begin{aligned}
& D(x, y, \sigma)=L(x, y, k \sigma)-L(x, y, \sigma) \\
& L(x, y, \sigma)=G(x, y, \sigma) * I(x, y) \\
& G(x, y, \sigma)=\frac{1}{2 \pi \sigma^{2}} e^{-\left(x^{2}+y^{2}\right)} / 2 \sigma^{2}
\end{aligned}
$$

where

$$
\begin{aligned}
& \mathrm{x}, \mathrm{y}=\text { image coordinates } \\
& \sigma=\text { scale }
\end{aligned}
$$

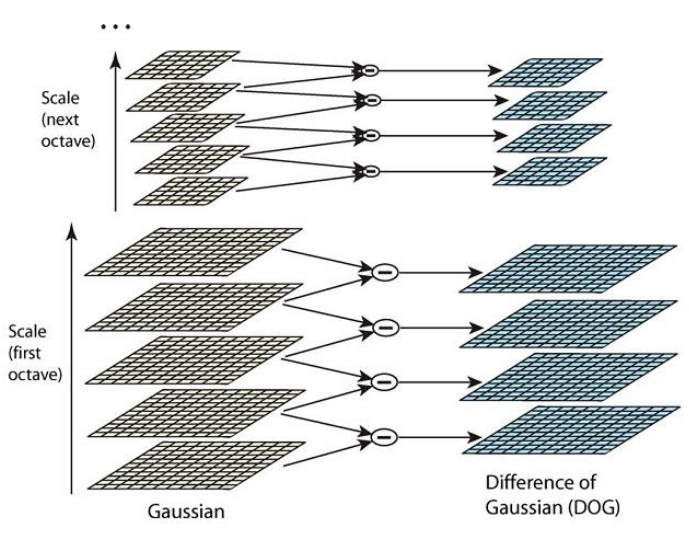

Figure 1. calculate DOG

and select the maxima and minima locations. Then, to make the position more accurate, we interpolate the data by using the quadratic Taylor expansion of the Difference-of-Gaussian scale-space function.

$$
D(x)=D+\frac{\partial D^{T}}{\partial x} x+\frac{1}{2} x^{T} \frac{\partial^{2} D}{\partial x^{2}} x
$$

If the offset of the keypoint is larger than 0.5 then we change the keypoint and calculate again or if the offset is small than 0.5 then we add the offset to the keypoint to get subpixel estimate of the keypoint.

Finally the keypoint is select among the candidate location based on their stability.

\subsubsection{DES CRIBE AND MATCH THE KEYPOINTS}

After those keypoint have been extracted, based on the local image gradient directions, assign one or more orientations .E.g.

$$
\begin{aligned}
& m(x, y)=\sqrt{(L(x+1, y)-L(x-1, y))^{2}+(L(x, y+1)-L(x, y-1))^{2}} \\
& \theta(x, y)=\tan ^{-1}\left(\frac{L(x, y+1)-L(x, y-1)}{L(x+1, y)-L(x-1, y)}\right)
\end{aligned}
$$

After calculate the orientation, set orientation histograms on $4 \times 4$ pixel neighborhoods with 8 bins each.Then compute the magnitude and orientation values of the samples in a $16 \times 16$ region around the keypoint so each histogram contains samples from a $4 \times 4$ subregion of the original neighborhood region. 
Furthermore, weighted the magnitudes by a Gaussian function with $\sigma$ equal to one half the width of the descriptor window. Finally the descriptor is extracted.

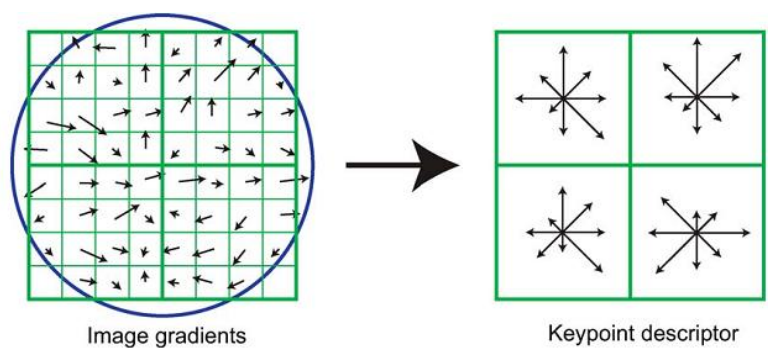

Figure 2. calculate the descriptor

In order to matching the corresponding image point, compare the two most similar keypoints of the stereo image. If the different is bigger than the threshold value then we believe is the right corresponding image point.

Here, to make matching it more efficient, we use k-d tree accelerate the process.

\section{- 2.2TIN PREDICT THE POTENTIAL} CORRES PONDING IMAGE POINT

After get the corresponding image points from SIFT matching, we use TIN (Triangulated Irregular Network) to predict to potential corresponding image point of the added point.

To predict the potential point, we use the points that in the left to build the TIN and set the $\mathrm{x}$ and $\mathrm{y}$ as the height of the corresponding points respectively. So we can predict the added point's corresponding image point's $x, y$ respectively.and use the initial corresponding image point to calculate the initial height.

\subsection{REGION-BASED LEAS T SQUARES MATCHING}

With the initial height, we can use the Region-Based least squares matching to calculate the accurate corresponding image point.

\subsubsection{THE RADIANT RELATIONSHIP}

As showed in the figure 3.the left image is the matching image and the right image is the matched image, $\mathrm{p}_{0}\left(\mathrm{x}_{0 \mathrm{c}}, \mathrm{y}_{0 \mathrm{c}}\right)$ is the added point and $\mathrm{p}_{1}\left(\mathrm{X}_{1 \mathrm{c}}, \mathrm{y}_{1 \mathrm{c}}\right)$ is the corresponding image point.

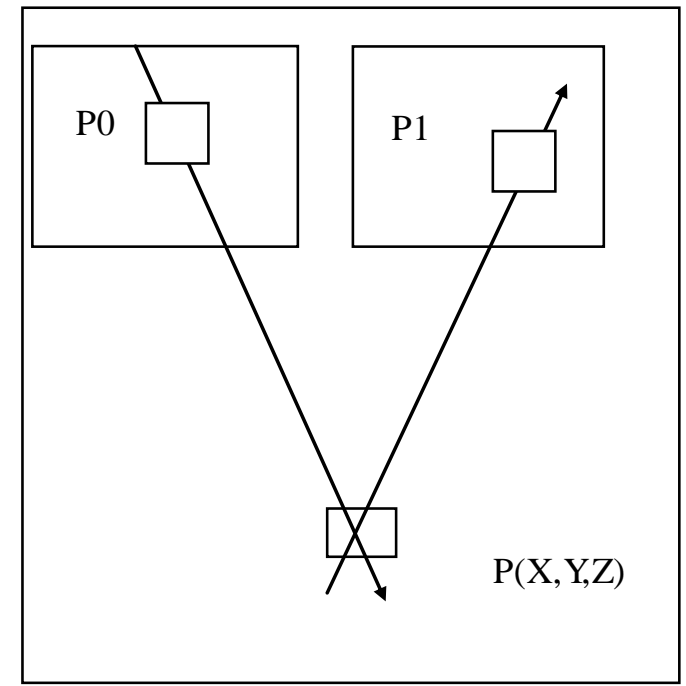

Figure 3.radiant relationship

In Region-Based least squares matching, firstly, we calculate three-dimensional coordinates position of the added point $\mathrm{P}(\mathrm{X}, \mathrm{Y}, \mathrm{Z})(\mathrm{ZHANG}$ Guo,2011). Then we calculate it back to the matched image. Without the consideration of random noise, the change of the radiation can be described as below.

$g_{0}\left(x_{0}, y_{0}\right)=h_{01}+h_{11} \cdot g_{1}\left(x_{1}, y_{1}\right)$

where

$g_{0}\left(x_{0}, y_{0}\right)=$ the gray in left image

$g_{1}\left(x_{1}, y_{1}\right)=$ the gray in right image

$h_{01}, h_{11}=$ the variates in radiation 
2.3.2THE GEOMETRICAL CONSTRAINT OF PROJECTION MODEL

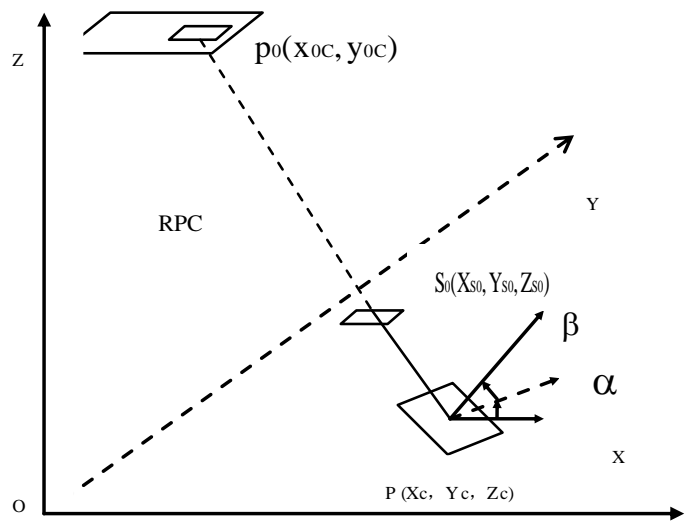

Figure 4. geometrical constraint of projection model figure 4 shows the projection relationship of the Region-Based least squares matching. In figure $2, \mathrm{p}_{0}$ is the added point we use rational polynomial coefficients (RPC)(ZHANG Guo,2010) to calculate the Coordinate $\mathrm{P}$ in the slope. Through $\mathrm{P}$ the normal line is decided by $(\alpha, \beta)$. And the slope can be described as fellow.

$$
\begin{aligned}
& a\left(X-X_{C}\right)+b\left(Y-Y_{C}\right)+c\left(Z-Z_{C}\right)=0 \\
& a=\cos \alpha \cos \beta \\
& b=\sin \alpha \cos \beta \\
& c=\sin \beta
\end{aligned}
$$

Here, in order to simulate the projection of single-center projection, we make a fake projection centre at the height of $\mathrm{Zs}_{\mathrm{s} 0}$ e.g $\mathrm{S}_{0}\left(\mathrm{Xss}_{\mathrm{s}}, \mathrm{Y}_{\mathrm{s} 0}, \mathrm{Z}_{\mathrm{s} 0}\right)$. As the distant is short, we regard the projection between the fake projection centre and point in the slope as parallel projection. So we can get the projection equation as fellow.

$$
\left.\begin{array}{l}
X=X_{s 0}+\lambda\left(X_{0}-X_{s 0}\right) \\
Y=Y_{s 0}+\lambda\left(Y_{0}-Y_{s 0}\right) \\
Z=Z_{s 0}+\lambda\left(Z_{0}-Z_{s 0}\right)
\end{array}\right\}
$$

where

$\left(X_{s 0}, Y_{s 0}, Z_{s 0}\right)=$ the coordinate of the fake projection centre

$\left(X_{0}, Y_{0}, Z_{0}\right)=$ one coordinate in the slope $\lambda=$ projection coefficient

With the consideration of radiant relationship and geometrical constraint of projection model the error equation is showed as fellow:

$v_{g 0}\left(s_{0}, l_{0}\right)=d h_{01}+g_{1}\left(s_{1}, l_{1}\right) \cdot d h_{11}+h_{11}\left(\frac{\partial g_{1}}{\partial s_{1}} d s_{1}+\frac{\partial g_{1}}{\partial l_{1}} d l_{1}\right)-\left(g_{0}\left(s_{0}, l_{0}\right)-h_{01}-h_{11} \cdot g_{1}\left(s_{1}, l_{1}\right)\right)$

in the error equation the variates are the height of the centre of the slope $Z_{C}$, direction angle $\alpha, \beta$, the variates in radiation $h_{01}, h_{11}$.

\section{EXPERIMENT AND RESULT}

To test the effect of this, here we use the stereo pairs in Lanzhou to product DEM the detail of data is show in below

\begin{tabular}{|l|l|l|l|l|}
\hline satellite & $\begin{array}{l}\text { Image } \\
\text { mode }\end{array}$ & date & $\begin{array}{l}\text { Incidence } \\
\text { angle }\end{array}$ & $\begin{array}{l}\text { Lat and } \\
\text { lon of } \\
\text { centre }\end{array}$ \\
\hline COSMO-SkyMed & HI & $2011-4-22$ & $24.7^{\circ}$ & $\begin{array}{l}\mathrm{N} 36.17^{\circ} \\
\mathrm{E} 103.93^{\circ}\end{array}$ \\
\cline { 2 - 6 } & HI & $2011-5-17$ & $41.0^{\circ}$ & $\begin{array}{l}\mathrm{N} 36.18^{\circ} \\
\mathrm{E} 103.84^{\circ}\end{array}$ \\
\hline \multirow{2}{*}{ TerraSAR-X } & $\mathrm{SM}$ & $2011-4-30$ & $28.7^{\circ}$ & $\begin{array}{l}\mathrm{N} 36.20^{\circ} \\
\mathrm{E} 103.81^{\circ}\end{array}$ \\
& & & & $\mathrm{N} 36.15^{\circ}$ \\
\cline { 2 - 5 } & $\mathrm{SM}$ & $2011-5-16$ & $44.4^{\circ}$ & $\mathrm{E} 103.81^{\circ}$ \\
\hline
\end{tabular}

Table 1 detail of data

After matching, we can get the image pair of corresponding image point 


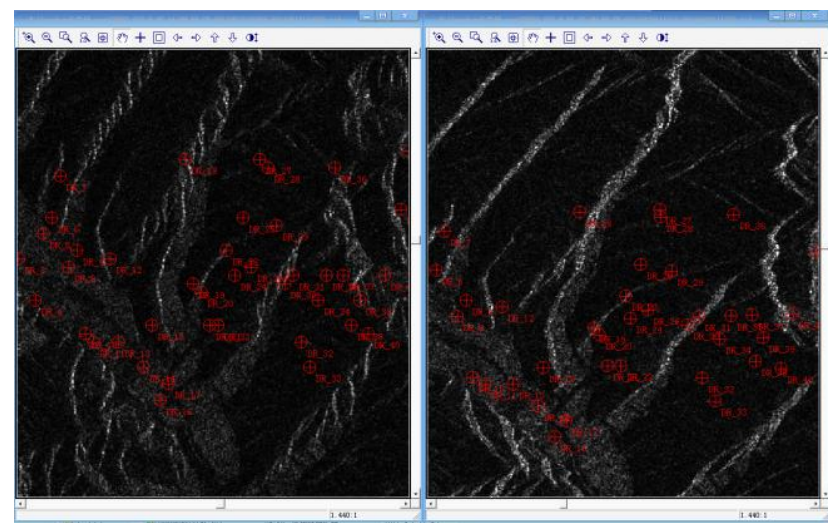

corresponding image point of COSMO-SkyMed

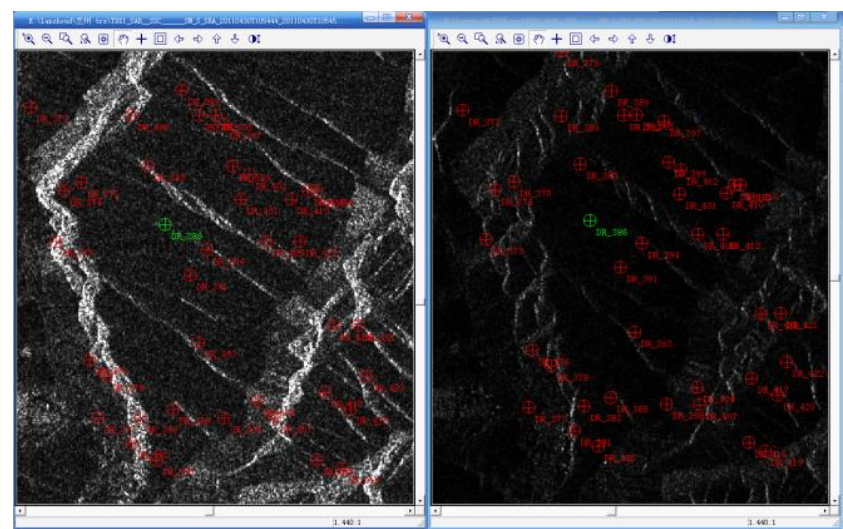

corresponding image point of TerraSAR-X

We compare the DEM produced by those corresponding image points with a 1:10000DEM

\begin{tabular}{|l|l|l|l|l|l|}
\hline COSMO-Sky Med & \multicolumn{1}{|c|}{$\begin{array}{r}1: 10000 \\
\text { DEM }\end{array}$} & $\begin{array}{c}\text { Residuals } \\
\text { of heght }\end{array}$ & \multicolumn{1}{|c|}{ TerraSAR-X } & $\begin{array}{c}1: 10000 \\
\text { DEM }\end{array}$ & $\begin{array}{c}\text { Residuals } \\
\text { of hight }\end{array}$ \\
\hline 1778.9 & 1777.2 & 1.7 & 1504.5 & 1497.4 & 7.1 \\
\hline 1794.2 & 1794.1 & 0.1 & 1788.7 & 1784.8 & 3.9 \\
\hline 1789.6 & 1782.1 & 7.5 & 1801.8 & 1806.6 & 4.8 \\
\hline 1783.9 & 1773.3 & 10.6 & 1809.2 & 1784.8 & 14.4 \\
\hline 1780.5 & 1773.3 & 7.2 & 1573.2 & 1577.6 & 4.4 \\
\hline
\end{tabular}

Table 2. accurate of DEM

\section{CONCLUSION}

This method is effect to match stereo SAR image, but in some area the result is not good enough .after analysis the data and algorithm, we realize that those situation happen because SIFT matching cannot get enough corresponding image points, so the initial height is not accurate enough to calculate the right point.

To avoid this kind of fault, in the future we need to try different filter to improve result of SIFT matching and try some different ways to forecast the initial height.

\section{REFERENCES}

Lowe,D.G. 1999. Object Recognition from Local Scale-Invariant Features.In International Conference on Computer Vision, Corfu, Greece, pp.1150-1157.

Lowe,D.G. 2004. Distinctive Image Features from Scale-Invariant Keypoints. International Journal of Computer Vision,60(2),pp.91-110.

ZHANG Guo,CHEN Tan,PAN Hongbo,JIANG Wanshou. 2011. Patch-based Least Squres Image Matching Based on Rational Polynomial Coefficients Model.Acta Geodaetica et Cartographica Sinica,40(5), pp. 592-597.

ZHANG Guo,FEI Wenbo,LI Zhen. 2010. Analysis and Test of the Substitutability of the RPC Model for the Rigorous Sensor Model of Spaceborne SAR Imagery [j]. Acta Geodaetica et Cartographica Sinica, 39(3),pp. 264-270. 